\title{
Respiratory diseases
}

\author{
D. Geraint James
}

Visiting Professor of Medicine, Royal Free Hospital, Pond Street, Hampstead, London NW3 2QG, UK

\section{Introduction}

Most of the references at the end of this review should be year-marked 1991 or 1992, with a few earlier ones if they are relevant to the discussion. Asthma is excluded because its worldwide importance merits a review of its own, and who better to do so than Professor Peter Barnes (see August 1992 issue of Postgrad Med J).

This review embraces techniques, Wegener's granulomatosis, occupational lung disease, infections, various pulmonary syndromes, neoplasia, granulomatous disorders and therapeutics. This year, therapeutics highlights the newest immunomodulators which are in use or about to become available. They provide an exciting glimpse of what lies ahead.

\section{Techniques}

\section{Bronchoalveolar lavage}

Bronchoalveolar lavage (BAL) has provided a helpful new window to the lungs. Changes in the cellular and humoral constituents of BAL fluid (BALF) have broadened our understanding of respiratory and multisystem disorders (Table I). It may be helpful in the differential diagnosis of sarcoidosis and fibrosing alveolitis for CD4 helper cells are prominent in BALF of active sarcoidosis whereas neutrophils are prominent in that of fibrosing alveolitis (Table II).

The BAL window permits new glimpses or even wider perspectives of asbestosis, cobalt lung, AIDS, pulmonary alveolar proteinosis (milky BALF), eosinophilic pneumonia, transplants (lung, liver and bone marrow), Crohn's disease, collagen vascular disease and Langerhan's histiocytosis. ${ }^{1}$

Correspondence: D.G. James, M.A., M.D., F.R.C.P.

\section{Thoracoscopy}

Thoracoscopy was widely used in the 1940s in the era of artificial pneumothorax collapse therapy for tuberculosis. It enabled adhesions to be severed and improve collapse of the tuberculous lung. Antituberculous chemotherapy made collapse therapy obsolete, but, unfortunately, this also led to the disuse of thoracoscopy. A recent plan from Montreal $^{2}$ should help to revive its use in the evaluation of patients with pleural disease, which remains undiagnosed after the usual initial investigations. Thoracoscopy done under local anaes? thesia is a rapid, safe and well-tolerated procedure with an excellent diagnostic yield. Biopsy specio mens of parietal pleura, obtained under direc vision, were studied by routine histology, mycobacterial culture, immunochemistry and electron

Table I A preponderance of certain cells in bronchoalveolar fluid may be a helpful diagnostic pointer

\begin{tabular}{ll}
\hline Cell & Disorder \\
\hline T4 helper & $\begin{array}{l}\text { Sarcoidosis } \\
\text { Beryllium disease }\end{array}$ \\
T8 suppressor & $\begin{array}{l}\text { Extrinsic allergic alveolitis } \\
\text { (Hypersensitivity pneumonitis) }\end{array}$ \\
& Talc \\
Smoker \\
Neutrophil & Fibrosing alveolitis \\
& AIDS \\
& Mineral dust \\
& Collagen disease \\
Eosinophil & Drugs \\
& Eosinophilic pneumonia \\
Mast cell & Bronchial asthma \\
Multinucleate giant & Sarcoidosis \\
Plasma & Asthma \\
Reed-Sternberg & Mubalt \\
Anaplastic & Hodgkin's \\
OKT6 staining & Lymphangitic carcinoma \\
\end{tabular}


Table II Bronchoalveolar lavage is helpful in the differential diagnosis of sarcoidosis and fibrosing alveolitis

\begin{tabular}{|c|c|c|}
\hline Feature & Sarcoidosis & Fibrosing alveolitis \\
\hline Dyspnoea & Mild & Severe \\
\hline Clubbing & No & Yes \\
\hline Added sounds & Insignificant & $\begin{array}{l}\text { Numerous end- } \\
\text { inspiratory }\end{array}$ \\
\hline Chest X-ray & Generalized & Lower zone \\
\hline Hilar adenopathy & Present & Absent \\
\hline $\begin{array}{l}\text { Honeycombing } \\
\text { CAT scan }\end{array}$ & Infrequent & Common \\
\hline Distribution & Central & Peripheral \\
\hline Changes & Linear & Cystic \\
\hline SACE & Increased & Normal \\
\hline BAL & $\begin{array}{l}\text { T4 cells } \\
\text { increased }\end{array}$ & Neutrophils \\
\hline Gallium scan & Positive & \pm \\
\hline Response to steroids & Good & Fair \\
\hline
\end{tabular}

$\mathrm{CT}=$ computed tomography; SACE = serum angiotensin converting enzyme; $\mathrm{BAL}=$ bronchoalveolar lavage.

microscopy. This technique led to the diagnosis of mesothelioma and various metastatic carcinomas, asbestosis, tuberculosis, rheumatoid arthritis, systemic lupus, chylothorax and haemothorax.

This is the decade of endoscopic cholecystectomy and keyhole surgery. Could this old and well-established technique give rise to endoscopic resection of parts of the lung? It is certainly being used for carbon dioxide laser treatment of bullous emphysema in California. ${ }^{3}$ Patients had advanced emphysema with severe pulmonary dysfunction and were too high a risk for conventional surgical bullectomy. In this study, there was significant objective improvement, which has persisted. Longterm follow-up will determine its precise value in this severely handicapped group of patients.

\section{Rapid diagnosis}

The demand for rapid viral diagnosis has increased in recent years particularly since antiviral chemotherapy has made important advances. The detection of pathogen-specific DNA after amplification with the polymerase chain reaction will revolutionize the whole science of microbiology. This will be a boon in the detection of respiratory viruses, which, thus far, have been difficult to identify by rapid methods. Respiratory syncytial virus causes winter childhood bronchiolitis, and detection of antigen by either immunofluorescence or enzyme immunoassay is reliable. ${ }^{4}$ Polyclonal antisera (Wellcome) against influenza viruses, parainfluenza and adenovirus group antigens are suitable for immunofluorescence testing. Another ingen- ious technique is fluorescent focus assay, which entails centrifugation of the respiratory specimen on a monolayer of tissue culture cells, incubation overnight, and then staining the cells with immunofluorescent antisera. This Australian review ${ }^{4}$ provides an exciting perspective of what we may learn to expect during the remainder of this decade. Read it now before it will be overtaken by continuing rapid advances 'in the field' and away from the reference laboratory.

A joint Anglo-Chinese study has facilitated the diagnosis of nasopharyngeal carcinoma by means of recombinant Epstein-Barr virus (EBV) proteins. ${ }^{5}$ Nasopharyngeal carcinoma, a tumour of epithelial origin, is common in the Chinese with an incidence of 15-30 per 100,000 per year. In Chinese populations it is the commonest tumour among men and the second most common among women. The immune response of patients with nasopharyngeal carcinoma to EBV antigens is diagnostic of the tumour. The EBV thymidine kinase was found to be the most sensitive predictor of nasopharyngeal carcinoma when used in a recombinant expression system. All serum samples analysed showed IgG and $\operatorname{IgA}$ antibodies to thymidine kinase whereas samples from infectious mononucleosis are negative. This technique is suitable for use in large-scale screening programmes for the early diagnosis of nasopharyngeal carcinoma.

The use of a rapid method of diagnosing toxoplasmosis by the polymerase chain reaction is a pointer to the future for all microbiology specimens, and this DNA probe is now established as a specific and highly sensitive means of detecting toxoplasmosis in a variety of specimens including serum, cerebrospinal fluid (CSF), bronchoalveolar fluid and biopsy material. ${ }^{6}$

Likewise with Pneumocystis carinii pneumonia, which can be diagnosed rapidly by DNA amplification of sputum and bronchoalveolar fluid. It is a highly sensitive and invaluable new technique.?

Even more important is the early diagnosis of tuberculosis by DNA amplification. It is more sensitive than bacteriological culture, and it is particularly recommended for the early diagnosis of tuberculous meningitis, tuberculous pleurisy and tuberculosis in human immunodeficiency virus (HIV)-positive patients. ${ }^{8}$

\section{A new bronchoscopic sign}

Pulmonary veno-occlusive disease (pulmonary vascular occlusive disease) is a rare disorder of unknown aetiology affecting children and young adults, who present with dyspnoea, signs of pulmonary hypertension, cyanosis, fine crepitations, and the chest X-ray must show bilateral patchy 
consolidation. In a recent case report, fibreoptic bronchoscopy showed a most unusual and previously unrecognized appearance. ${ }^{9}$ The trachea and main bronchi were normal but the lobar and segmental bronchi showed intense hyperaemia with vascular engorgement in the form of bright red longitudinal streaks. The sharp demarcation between normal main bronchi and the very red lobar and segmental bronchi is due to the venous drainage of the bronchial tree. The trachea and main bronchi drain into the bronchial veins, which were presumably normal. The more peripheral bronchi drain into the pulmonary veins, which were congested by the occlusive process.

\section{Langerhans' pulmonary dendritic cells}

Paul Langerhans (1847-1888) was born and studied medicine in Berlin and became professor of pathology in Freiburg. He is remembered by the islets of Langerhans and posthumously by Langerhans' pulmonary dendritic cells. They are found in the peribronchial connective tissue and in the alveolar parenchyma. As in the skin, they are derived from less sophisticated indeterminate dendritic cells. It would be interesting to know what mediators convert these dendritic cells into sophisticated Langerhans' macrophages, which are frequently seen in zones of alveolar hyperplasia and alveolar metaplasia induced by tobacco or other inflammatory insults. The French ${ }^{10}$ remind us that Langerhans' pulmonary macrophages are well worth studying for they have a potent capacity for presenting antigen to lymphocytes, and thereby a significant role in the pulmonary immune response and in the pathogenesis of histiocytosis $X$, now renamed Langerhans' granulomatosis or Langerhans' cell histiocytosis, and in pulmonary lymphomas. Langerhans' $X$ or HX cells have a cleaved nucleus with a finely dispersed chromatin pattern within an eosinophilic cytoplasm. Electron microscopy reveals pentalaminar structures within this cytoplasm.

These cells are described under the heading Techniques because their prominence and importance are being increasingly recognized and they will repay further study to analyse their role in systemic diseases.

\section{Mediastinal sonography}

Wernecke, a Munster radiologist, pioneered suprasternal and parasternal mediastinal sonography, and has now applied this technique to guidance of percutaneous mediastinal tumour biopsy. ${ }^{11}$

The essential criterion for selection of patients is the availability of an avascular approach to the mediastinal tumours, so sonographic guidance is mainly suitable for the biopsy of anterior medias- tinal and supra-aortic tumours. Accidental puncture of the mediastinal vessels, which is not uncommon under fluoroscopic guidance, can be largely $\$$ avoided with exact computed tomography guided $\stackrel{\varrho}{c}$ planning of the biopsy route. In addition, the exact location of the needle tip in the target lesion can be $\stackrel{5}{9}$ reviewed before biopsy. The specimens obtained were lymphoma, bronchial carcinoma, metastases, $\frac{}{\sigma}$ carcinoid, thyroid cyst and haematoma. Its value is $\frac{\bar{\omega}}{\overrightarrow{2}}$ to distinguish a primary mediastinal tumour $\stackrel{\varnothing}{\Omega}$ requiring surgery from systemic lymphatic malignancy. This technique is simple, rapid and accurate, and provides yet another window to the lungs.

\section{Intravascular ultrasound}

Transthoracic and transoesophageal echocardio- 3 . graphy can reveal evidence of pulmonary embolism $\dot{\infty}$ but only to the mainstem and central parts of the left and right pulmonary arteries. Intravascular ultrasound probes the periphery of the pulmonary $\overrightarrow{\vec{\sigma}}$ vascular bed. ${ }^{12} \mathrm{~A}$ catheter is introduced via a $\circ$ guidewire into the main trunk of the pulmonary artery and advanced into the left and right pul- monary trunk. It could be advanced into vessels smaller than $1 \mathrm{~cm}$ in diameter. The catheter is connected to an ultrasound device and the sonograms are stored on videotape. It should be poss $\vec{\theta}$ ble to use an indwelling catheter to monit\& $N$ thrombolytic therapy in pulmonary embolism.

\section{Apoprotein-A concentration in sputum}

Pulmonary alveolar proteinosis (PAP) is a disorder characterized by accumulation of surfactant materials in alveoli. It may be recognized by bronchoalveolar lavage for the wash is milky due to intra-alveolar accumulation of periodic acid-Schiff positive proteinaceous material. The predominant phospholipid-associated glycoprotein in pulmonary surfactant is surfactant aprotein A (SP-A). This may be measured in sputum using monoclonal antibodies to SP-A.

Sputum concentrations of SP-A were found in this Japanese study to be 400 times higher in PAP than in controls. ${ }^{13}$ This simple non-invasive technique must be an attractive alternative to any invasive procedure.

\section{Wegener's granulomatosis (WG)}

In last year's review, we reported that Friedrich Wegener lived in happy retirement in Lubeck, Germany. This year it is sad to report his death on 9 July 1990, aged 83 years. ${ }^{14} \mathrm{He}$ lived to see two remarkable advances in the disorder which bears his name. He lived to see the introduction of a most helpful diagnostic blood test; anti-neutrophil cyto- 
plasmic antibodies (ANCA) are identified in serum of patients with Wegener's granulomatosis by immunofluorescence microscopy using alcoholfixed normal human neutrophils. He also derived considerable joy from the second advance, namely treatment of the disease. When he first described the disorder in 1936 it carried a $100 \%$ mortality. In the succeeding 50 years, he witnessed the introduction of corticosteroids, cyclophosphamide and antibiotics, all of which have combined to reduce the mortality to a more manageable $5 \%$. He was thrilled to feel that earlier diagnosis by a noninvasive procedure led to earlier effective treatment and a substantial improvement in prognosis.

The National Institutes of Health, Bethesda, have recently analysed the pulmonary pathological features in 87 open lung biopsies from 67 patients with WG, and illustrated the wide variation and broad spectrum of pathological changes observed. ${ }^{15}$ The three useful diagnostic criteria were parenchymal necrosis, vasculitis, and granulomatous inflammation accompanied by an inflammatory infiltrate of plasma cells, neutrophils, lymphocytes, histiocytes and eosinophils. Parenchymal necrosis comprised neutrophilic microabscesses or areas of geographic necrosis surrounded by palisading histiocytes and giant cells. Granulomas were poorly formed with scattered giant cells. Vasculitis was almost always present in arteries and veins; commonly necrotizing and granulomatous with cicatricial changes and, infrequently, fibrinoid necrosis. Minor changes were alveolar haemorrhage, bronchiolitis obliterans with organizing pneumonia (BOOP) and nodular interstitial fibrosis.

Despite a diagnostic blood test and histological features of a granulomatous vasculitis there continue to be diagnostic difficulties. This is evident by a recent case report of a patient with a $T$ cell lymphoma of the lung mimicking WG. ${ }^{16}$ The bronchial biopsy was compatible with WG, ANCA was positive, and the initial response to cyclophosphamide and prednisolone was most encouraging. However, 5 months after presentation a repeat bronchial biopsy revealed lymphoma and underlined the dictum 'all that glisters is not Wegener's granulomatosis'.

\section{Occupational lung disease}

Asbestos

After deposition of asbestos, the initial lesion is an accumulation of alveolar macrophages in the alveolar ducts and peribronchial region adjacent to the terminal respiratory bronchiole. This is thickened by interstitial macrophages and later by fibroblasts; and type 1 alveolar cells are injured by this process and by mediators released by macrophages as they phagocytose free asbestos fibres. These chemical mediators include fibronectin, platelet-derived growth factor, alveolar macrophage-derived growth factor and insulin-like growth factor. What starts insidiously and progresses inexorably ends lethally with the development of interstitial and pleural fibrosis, mesothelioma, lung cancer and gastrointestinal malignancy. These costly diseases are well-reviewed in an authoritative recent state of the art. ${ }^{17}$ The processes of inflammation, fibrosis and carcinogenesis appear to be closely interwined; proto-oncogenes are upregulated in activated alveolar macrophages from fibrotic lungs. Furthermore asbestos can transfect DNA into cells. Cigarette smoking prepares a highly metaplastic bronchial epithelium and so provides co-carcinogenicity.

Circumscribed pleural plaques and diffuse pleural thickening, collectively termed pleural fibrosis, is the most common radiographic manifestation of asbestos exposure. Its development is dependent on the cumulative dose of asbestos exposure and the elapsed time since the first exposure. Autopsy studies and computed tomographic (CT) scans suggest that the standard chest radiograph only identifies about $50-80 \%$ of the pleural plaques that are actually present. ${ }^{18}$ This is disclosed by a recent survey at the London Chest Hospital, in which CT scanning was compared with chest radiography in 20 patients with asbestos-induced pleural disease. ${ }^{19} \mathrm{CT}$ scan always reveals involvement in the paravertebral area. CT demonstrated discrete plaques in $95 \%$ of patients but these were only identified in $59 \%$ of patients by chest radiography. Diffuse pleural thickening was evident on the CT scan in all patients and in $70 \%$ on the radiograph. CT and chest radiograph scores inversely correlated with all lung function measurements except $\mathrm{KCO}$. Increasingly extensive pleural disease is associated with increasingly severe impairment of lung function. The superiority of CT may be important for those claiming state compensation for asbestos-induced disease.

\section{Clay dye pneumoconiosis}

This benign pneumoconiosis among rush mat workers is only seen in Japan. The rush mat (tatami and fancy mat) is treated with a clay dye which contains quartz, chlorite, kaolin and sericite, with a high free silica content. The rush is dipped into a $40 \%$ colloidal soluton of clay dye, which imparts to the mat a silver-green hue and also a certain degree of elasticity essential for matting purposes. ${ }^{20}$ In the course of 10 years, the chest X-ray reveals small round opacities less than $1.5 \mathrm{~mm}$ in diameter, distributed throughout the lungs. The histopathology is of small pneumoconiotic weakly fibrotic 
nodules composed of macrophages phagocytosing or attached to dust particles around peripheral perivascular and alveolar regions. The disease process shows fine dust foci but only a weak tendency to progress to fibrosis, possibly because the clay coats free silica thereby reducing fibrogenic potential.

\section{Hut lung}

Occupational lung disease is more common in men but clay dye pneumoconiosis is frequent in Japanese women workers, and hut lung is a domestically acquired pneumoconiosis in women from the Transkei district of South Africa. ${ }^{21}$ Its alternative name is Transkei silicosis. It is due to silica particles inhaled when they are hand-grinding maize between rocks in poorly ventilated huts. The maize kernels are ground between an oblong top rock, which is handled, and a large base rock on the ground. Twenty-five women were studied by Cape Town workers, who report that most women were symptom-free but radiological findings ranged from a military pattern to extensive fibrosis resembling progressive massive fibrosis. Autopsies on two patients who died from respiratory failure confirmed features of silicoanthracosis. This domestically acquired pneumoconiosis is not only due to maize grinding but also due to smoky woodfuelled stoves in poorly ventilated huts; hence the more appropriate term 'hut lung'.

\section{SWORD}

This stands for Surveillance of $W$ ork-related and Occupational Respiratory Disease. ${ }^{22}$ Members of the British Thoracic Society set up a reporting scheme for this endeavour in which yellow cards are returned concerning any patients with possible occupational lung disease. The SWORD scheme will provide an important base of information, from which we may track trends and monitor the efficacy of preventive measures in respiratory disease.

\section{Papworth Hospital, Cambridge}

The Tim Higgenbottam-John Wallwork team maintain standards of excellence in their pioneering human lung transplantation programme, which is still in its infancy compared with other forms of organ transplantation. Improved surgical technique, preservation of donor organs, and cyclosporin have been important advances, but these advances have been offset by acute pulmonary infections, fatal obliterative bronchiolitis, and chronic rejection. Chronic rejection is the major cause of death of long-term survivors of heart-lung transplantation. In a Papworth series of 61 patients who had received heart-lung transplants, 7 patients developed chronic rejection, 4 died with extensive obliterative bronchiolitis, 12 have shown lung fibrosis, and opportunistic lung infections resulted in pneumonia on 19 occasions. ${ }^{23}$ Herpes simplex virus infection is sufficiently frequent to warrant prophylactic acyclovir for the first 2 months after surgery and at times of augmented immunosuppression. ${ }^{24}$

Early detection and prompt treatment of acute rejection is an essential step in the prevention of the crippling obliterative bronchiolitis of chronic rejection. Close monitoring of lung function with regular routine and diagnostic transbronchial lung biopsy enables accurate diagnosis of acute rejection and may have lessened the incidence of chronic rejection. ${ }^{25}$

Papworth has a wider range of interests than just the transplant programme for they have also investigated impairment of endothelium-derived relaxing factor (EDRF) in the pulmonary circulation in chronic hypoxic lung disease. Endotheliumdependent pulmonary artery relaxation in vitro is impaired in arteries from patients with end-stage chronic obstructive lung disease compared with control pulmonary arteries from patients undergoing lobectomy for lung carcinoma. The response of all vascular rings to the endothelium-dependen vasodilators, acetylcholine and adenosine diphoso phate, were studied immediately after lung ex? cision. The impairment may contribute to the development of pulmonary hypertension in chronic hypoxic lung disease. ${ }^{26}$

Endothelin-1, a potent endothelium-derived vasoconstrictor peptide, has been studied in Quebec, and, in particular, its role in pulmonary hypertension, by measuring its concentration in arterial and venous plasma. ${ }^{27}$ Patients with pulmonary hypertension were found to have substantial alterations in plasma immunoreactive endothelin-1, reflecting changes in net release or clearance by the lung. In patients with primary pulmonary hypertension, the high levels in arterial compared with venous plasma suggest pulmonary production of endothelin-1 which may contribute to pulmonary vascular resistance.

\section{Tuberculosis}

\section{Can we eradicate tuberculosis?}

In April 1989, Dr Louis Sullivan, US Secretary of Health and Human Services announced his endorsement of the goal of eliminating tuberculosis from the United States by the year 2010. This laudable ambition has led to an Advisory Committee for the Elimination of Tuberculosis and its joint 
curriculum with the American Thoracic Society and the Centers for Disease Control, Atlanta; all greatly respected bodies. The most recent document developed by these groups is an excellent 'Core Curriculum on Tuberculosis', obtainable from Technical Information Services, Center for Prevention Services, Atlanta, Georgia. ${ }^{28}$ It encompasses epidemiology, transmission and pathogenesis of tuberculosis, diagnosis, preventive therapy and treatment. An estimated 10 million persons in the USA are infected and about $90 \%$ of new cases arise from this already infected pool. In 1986 , after three decades of steadily decreasing tuberculosis morbidity, there was a turnaround increase in annual tuberculosis morbidity largely due to patients with acquired immunodeficiency syndrome (AIDS), but also due to medically underserved low-income populations and AfroAsian and Latin American migrants. Other highrisk groups who particularly need to be screened are alcoholics, intravenous drug users, residents of long-term care facilities (including prisons) and some health care workers. Much of this is already well accepted but it needs to be restated as tuberculosis soars in New York. ${ }^{29}$ Its prevalence in this city in 1990 was 46 cases per 100,000 , which is five times the national average. Moreover a casecontrol survey has shown nosocomial spread of drug-resistant tuberculosis in some of the city's hospitals. This may lead to the resurrection of tuberculosis sanatoriums in one of the most sophisticated cities in the most productive country in the world. There is similar frightening emergence of drug-resistant tuberculosis in drug addicts in Italy ${ }^{30}$ The global toll of tuberculosis is worldwide, a fact emphasized by recent figures of the Chief Medical Officer, Tuberculosis Unit, World Health Organisation. ${ }^{31}$ In 1990 , there were 8 million new cases of tuberculosis in developing and industrialized countries; $95 \%$ in the former and $5 \%$ in the latter. The worldwide pool of infected persons is 1,722 million, nearly one-half living in SouthEast Asia and the Western Pacific. Africa has an incidence of 272 cases per 100,000 . The incidence of tuberculosis in Hong Kong is 124 per 100,000 per annum, which is considerably more than in the white population in the UK. This has led to a warning that clinicians must be alerted to the possibility of tuberculosis, particularly its extrathoracic form, in migrants from Hong Kong when the lease back occurs in $1997 .^{32}$

The Medical Research Council in conjunction with the Hong Kong Chest Service and the Singapore Tuberculosis Service have recently updated their results of the chemotherapy of tuberculosis. The conduct of these continuing trials are elegant, and their design continues to command world respect. They are recommended reading in their entirety. ${ }^{33,34}$

\section{Mycobacterium avium-intracellulare (MAI)}

Organisms of this complex comprise two closely related species, $\boldsymbol{M}$. avium and $\boldsymbol{M}$. intracellulare. They have little virulence in the normal host, and chest physicians used to recognize them as an uncommon cause of pneumonia in patients with chronic lung disease. Those were the days before AIDS. They are now of considerable clinical importance in the AIDS population. By $31 \mathrm{De}-$ cember 1990, there were 12,202 cases of disseminated non-tuberculous mycobacterial infection among the 161,073 patients with AIDS reported to the Atlanta Centers for Disease Control. ${ }^{35} M$. avium complex, and also cytomegalovirus infection, become apparent when cell-mediated immunity is considerably depressed with CD4 + counts reduced to 100 or less cells $/ \mathrm{mm}^{3}$. Clinical presentations include fever, night sweats, weight loss (all suggesting toxicity by tumour necrosis factor), pneumonia and endobronchial lesions as well as several extrathoracic lesions. Antimycobacterial agents include amikocin, ethambutol, streptomycin, ciprofloxacin and clofazimine. Horsburgh's authoritative review should be consulted for detailed management of this fatal disorder. ${ }^{35}$

\section{Fungal infection}

The Journal of Antimicrobial Chemotherapy has produced an excellent supplement on developments in the management of fungal infection..$^{36}$ It contains succinct accounts of invasive pulmonary aspergillosis, rhinocerebral mucormycosis, pulmonary cryptococcosis, new approaches to serodiagnosis, and the advent of fluconazole and itraconazole. The recognition of polyvalent and monoclonal antibodies to the major fungal pathogens has led to the marketing of diagnostic kits for the detection of circulating antigens in body fluids. Monoclonal antibodies to cell wall and cytoplasmic components of Cryptococcus neoformans and Histoplasma capsulatum have been described. Latex particle agglutination and double antibody liposomal-based formats are being marketed for systemic candidosis and invasive aspergillosis. Chromosomal DNA probes may lead to the detection of fungal elements in clinical specimens. This supplement is worth consulting in its entirety.

Invasive candidiasis is a major nosocomial infection that is difficult to diagnose. It has now been found that the presence of candida enolase in the blood is a novel marker for invasive candidiasis. It appears to be a useful indication of deep infection in patients with cancer and neutropenia; it complements the diagnostic usefulness of blood cultures. ${ }^{37}$ The British Society for Antimicrobial Chemotherapy Working Party have set out the 
indications for, and interpretation of, the tests that can be done with the major antifungal drugs in clinical use at present. ${ }^{38}$ They pay particular attention to flucytosine, ketoconazole, fluconazole and itraconazole. They set out sensitivity tests, assays of serum concentration, and regimens for administration of flucytosine in renal impairment, linking the dose with creatinine clearance. This is the type of article that will help the chest physician when he is faced with a respiratory mycosis.

\section{BOOP}

The name has stuck in the international literature so we must learn to recognize it for it is steroid sensitive, treatable and reversible. It stands for bronchiolitis obliterans organizing pneumonia (BOOP), which envelops two or perhaps even more conditions, but particularly cryptogenic organizing pneumonia and obliterative bronchiolitis. ${ }^{39,40}$ The French $^{41}$ recognize BOOP as a clinico-pathological entity with a well-recognized morphogenesis. Diffuse alveolar injury leads to basement membrane denudation and intra-alveolar exudate of fibrinogen, immunoglobulins, coagulation factors and fibronectin. Interstitial fibroblasts migrate into alveoli through denuded gaps in the epithelial basement membrane. The final step is the secretion of collagens I, III, V and fibronectin by fibroblasts forming a loose connective tissue fibrosis, which is surprisingly reversible. The diagnosis is confirmed by lung biopsy and it is usually steroid-responsive. It may be associated with primary biliary cirrhosis, the CREST syndrome and digital vasculitis and it may well overlap with various connective tissue disorders. Within the orbit of these overlap syndromes is the association of cryptogenic organizing pneumonitis with rheumatoid arthritis. ${ }^{42}$ The French ${ }^{43}$ have undertaken a wide survey of pleuropulmonary disease in rheumatoid arthritis, employing lung function tests, bronchoalveolar lavage and high resolution computed tomography. In this series nearly $50 \%$ of patients with rheumatoid arthritis had pleuropulmonary manifestations. This was particularly evident in men with subcutaneous nodules and positive rheumatoid factor. Bronchiolitis obliterans induced by penicillamine was the most severe iatrogenic feature, but they also observed hypersensitivity pneumonitis related to low dose methotrexate.

If you, dear readers, have reached thus far on BOOP, then you should take two further interesting academic steps. First, enjoy the Case Records of the Massachusetts General Hospital concerning a 68 year old man admitted with respiratory symptoms and an abnormal chest X-ray. Don't cheat by looking for the diagnosis at the end of the article. Just work out, step by step, your British management of this American patient. ${ }^{44}$ When you have done so you are nearly home and that means $\bar{z}$ reading Geddes' splendid editorial summarizing all $\stackrel{\mathbb{Q}}{\complement}$ views on BOOP and COP. ${ }^{45}$ It brings it all into $C$. perspective for another year or so. The fact that the $\overrightarrow{\vec{F}}$ same pattern of events occurs as a result of infections, drugs and connective tissue disorders suggests that it is not a single clinicopathological $\frac{\bar{\sigma}}{\bar{N}}$ entity but rather one way in which the lung mounts $\frac{\sigma}{\nabla}$ an inflammatory response to a range of different $\stackrel{\varnothing}{\varrho}$ insults. The term BOOP is probably best restricted $\%$ to those cases of unknown cause. ${ }^{45}$

\section{What shall we call this syndrome?}

The title of the article is too cumbersome for everyday use but the chest physician should be $\infty$ aware of it in women aged 45 years or so with pulmonary fibrosis and various multisystem . signs. ${ }^{46}$ These include Raynaud's phenomenon, polyarthralgia, polymyositis, sclerodactyly, keratoconjunctivitis sicca, dysphagia, facial telangiectasis, hepatitis, subcutaneous calcinosis, tendonitis and clubbing. The majority of patients have a greatly elevated sedimentation rate, raised serum immunoglobulins, and a third have a positive rheumatoid factor. The common laboratory fince ing in this series was the presence of autoantibodie against aminoacyl-tRNA synthetase; either hist dyl-tRNA synthetase (anti-Jo 1) or alanyl-TRNA $\vec{A}$ (anti-PL12) or threonyl-tRNA (anti-PL7) synthetase. The most important clinical determinant of outcome in this group of patients was the severity of the interstitial pulmonary disease. Five patients had transbronchial lung biopsies which showed features of interstitial lung fibrosis, two patients had chronic inflammatory cell infiltrates and one patient had evidence of active alveolitis with infiltrations of polymorphonuclear cells. No biopsy showed evidence of vasculitis. Various treatments included prednisolone, azathioprine, cyclophosphamide, methotrexate, cyclosporin and total body irradiation.

\section{Neoplasia}

\section{Non-small cell lung cancer (NSCLC)}

A whole volume of the US Current Problems in Cancer is devoted to non-small cell lung cancer, comprising squamous, large cell and adenocarcinoma, and forming $75 \%$ of lung cancer in the United States. ${ }^{47}$ Genetic mutations in dominant oncogenes such as $\mathrm{K}$-ras, loss of genetic material on chromosomes $3 \mathrm{p}, 11 \mathrm{p}$ and $17 \mathrm{p}$, and deletions or mutations in tumour suppressor genes such as rb and $\mathrm{p} 53$ have been documented in these tumour cell 
lines. NSCLC arises in a setting of bronchial metaplasia and dysplasia due to cigarette smoking and other pollutants. The prognosis is based on extent of tumour dissemination, ambulatory state and weight loss. Stages I and II NSCLC are confined within the pleural reflection, Stage III has extended through the pleura or metastasized to ipsilateral or subcarinal lymph nodes and Stage IV is characterized by distant metastases. Stages I and II are managed by surgical resection with approximate 5-year survival of $45 \%$ and $25 \%$ respectively; Stage III by a combination of resection and radiotherapy with a 5 -year survival of $15 \%$. Stage IV NSLC has a 5-year survival of less than $1 \%$ despite chemotherapy.

Growth hormone is a secretory product of some primary bronchial neoplasms and has been associated occasionally with the development of hypertrophic pulmonary osteoarthropathy and acromegaly. It may also be associated with finger clubbing. ${ }^{48}$ Increased plasma growth hormones levels correlated with clubbing; following chemotherapy in one patient hormone levels fell to normal and clubbing completely resolved. Further studies are needed to decipher whether the hormone is secreted by the tumour cells or the surrounding lung or from the pituitary after its stimulation by growth hormone releasing hormone.

There is renewed interest in the expression of $\mathrm{ABO}$ blood-group antigens in relation to the development of lung cancer. Expression of bloodgroup antigen $\mathbf{A}$ in tumour cells is an important favourable prognostic factor in patients with NSCLC. ${ }^{49}$ The authors of this report suggest that this factor should be taken into account when stratifying patients in future clinical trials to evaluate the efficacy of post-operative adjuvant chemotherapy. Patients who are negative for blood-group antigen $\mathrm{A}$ seem to merit aggressive therapeutic approaches in view of their poorer prognosis; perhaps chemotherapy and irradiation as an alternative to immediate surgery.

The Belgians have applied meta-analysis on studies of chemotherapy of NSCLC, involving a total of 6,247 patients. Polychemotherapy had a significantly better response rate than monotherapy, and the best results were associated with cisplatin, vindesine, vinblastine, mitomycin $\mathrm{C}$ and ifosfamide.$^{50}$ These differences were most obvious in those with limited rather than disseminated disease.

Workers at Yale University have tried to overcome the bleak outcome of Stage IIIa NSCLC by pre-operative (neoadjuvant) chemotherapy in order to decrease tumour bulk so that inoperable patients become surgical candidates. ${ }^{51}$

\section{Lymphoma}

A joint Anglo-American study of AIDS-related non-Hodgkin lymphoma analysed cases reported to the Centers for Disease Control, Atlanta, up to 30 June 1989. During this period 97,258 AIDS cases were reported, of whom $2,824(2.9 \%)$ had non-Hodgkin lymphoma: a condition that was about 60 times more common in AIDS patients than in the general US population. ${ }^{52}$ It was twice as common in whites as in blacks and in men as in women. It was most common in patients with haemophilia or clotting disorders and least common in those born in the Caribbean or Africa who had acquired HIV by heterosexual contact. Infectious agents, particularly Epstein-Barr virus, are associated with lymphoma in these immunosuppressed patients. The most difficult question is why Burkitt's lymphoma is commonly associated with HIV infection but not with other types of immunosuppression.

The European Lymphoma Co-operative Group have studied 772 patients with early-stage Hodgkin's disease, and assessed the value of an elevated $(>30 \mathrm{~mm} / \mathrm{h})$ Westergren erythrocyte sedimentation rate (ESR) for predicting early relapse and survival after therapy in patients with clinical Stage I or II Hodgkin's disease. An unexplained elevated ESR after therapy, especially modern radiotherapy, strongly suggests the presence of aggressive and resistant Hodgkin's disease. An elevated ESR is predictive of early relapse and poor prognosis; its presence justifies early aggressive therapy. ${ }^{53}$

Another test which provides useful prognostic information in low-grade lymphoma is the serum $\beta-2$ microglobulin $(\beta-2 \mathrm{M})$ level. It has been evaluated in 80 previously untreated Stage I to IV patients in Houston. ${ }^{54} \beta-2 \mathrm{M}$ is a low molecular weight polypeptide, the light-chain molecule of the major histocompatibility complex class 1 antigens. It is found on the membrane surface of almost all nucleated cells, and its membrane turnover is the principal source of $\beta-2 M$ in the blood and body fluids. The complete remission rate for patients with $\beta-2 \mathrm{M}$ level of $3 \mathrm{mg} / \mathrm{l}$ or greater was $36 \%$ compared with $71 \%$ for those with a level of less than $3 \mathrm{mg} / \mathrm{l}$. The mechanism is possibly that tumour cells release $\beta-2 M$ through membrane turnover. A similar helpful prognostic value is noted in myeloma, in which patients with the highest values have the shortest survival.

\section{Sarcoidosis}

Last year's review of respiratory diseases did not include sarcoidosis. As a result of this omission, we received several letters enquiring whether we had lost our taste and zest for this obsession of ours. 
Not so. This year has much more to include for in September 1991 was held the XII World Congress on Sarcoidosis in Kyoto, Japan, organized under the auspices of the World Association of Sarcoidosis and Other Granulomatous Disorders (WASOG). The Proceedings of this Congress will appear as a special supplement of the journal, Sarcoidosis. ${ }^{55}$ Indeed, as you read this article so will the supplement be available to update you on what sarcoidologists from 23 countries think about sarcoidosis and other granulomatous disorders such as Wegener's granulomatosis, summertime hypersensitivity pneumonitis, eosinophilic granuloma and occupational granulomatoses.

Sarcoidosis is published in Milan, Italy, in English every March and September. Each issue contains about 86 pages of articles on all aspects of sarcoidosis and other disorders which mimic it. It is also a thesaurus or treasure trove of information concerning conferences, events and news around the world.

An ambitious enterprise of the Kyoto conference was an epidemiological survey of sarcoidosis in 16 cities around the world. It analysed 2,702 new cases seen during 1981-1985, with women comprising $62 \%$ of the total. The age at presentation was in the $20-50$ year range in $78 \%$ of this series. Histological confirmation of the diagnosis is now frequently obtained by fibreoptic bronchoscopy and transbronchial biopsy. Open lung biopsy, scalene node and mediastinal lymph node biopsy are no longer popular. All patients in this series had intrathoracic sarcoidosis. The tempo of the disease varies with the community in which it is studied. The black population in the US and the West Indians in London often have severe, extensive and crippling disease whereas it is mild and with a high spontaneous rate of resolution in the Irish. Just as London sees much sarcoidosis among its migrants from the West Indies so does Paris see it in its migrants from Martinique, and New York City in its Puerto Rican-born population. Is this due to genetic predisposition or environmental factors confronting susceptible individuals migrating from a rural to an urban community? Is the breeding ground for sarcoidosis in their native homeland or within the sophisticated new world in which they adapt themselves? It may be a bit of both worlds.

\section{The road from granuloma to fibrosis}

Antigenic invasion by micro-organisms or chemicals or cancer is met by a granulomatous inflammatory response involving close interplay between activated macrophages bearing increased expression of major histocompatibility (MHC) Class II molecules and CD4 + lymphocytes. These CD4 cells help to initiate a cascade of chemotactic factors including interleukins 1 and 2 (IL-1 and $\cong$ IL-2), macrophage-activating and macrophage- $z$ inhibiting factors and gamma interferon. The $\stackrel{\varnothing}{\varrho}$ majority of T-cells bear the T-cell receptor (TCR) $\stackrel{.}{.}$ $\alpha \beta$ complex but a very small subpopulation bear a $\overrightarrow{\vec{F}}$ distinct TCR composed of gamma and delta $\stackrel{?}{+}$ subunits in lymphoid organs and skin. This small subpopulation of TCR $\gamma \delta$ lymphocytes is prom- 음 inent in leprosy, leishmaniasis and sarcoidosis, all $\frac{\tilde{\sigma}}{\bar{\sigma}}$

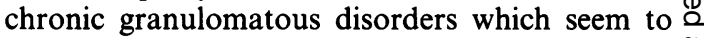
respond to mycobacterial stimulation. ${ }^{56}$

What of the lymphokines involved in this $\overrightarrow{0}$ granulomatous response? Pulmonary alveolar macrophages harvested in bronchoalveolar fluid of $\vec{\omega}$ sarcoidosis patients release tumour necrosis factor in vitro in response to Escherichia coli endotoxin $\underset{8}{0}$ lipopolysaccharide and spontaneously ${ }^{56,57}$ Those 3 . who have lived through the long history of claims $\infty$ for mycobacteria or their mucopolysaccharides as $\vartheta$ the causes of sarcoidosis will now recall, amongst $-v$ others, the pine pollen theory of sarcoidosis. Pine $\vec{\partial}$ pollen was acid-fast staining and resembled the wax fraction of the tubercle bacilli. Have we arrived back in square one of the aetiology of sarcoidosis? $?^{58} \overrightarrow{-}$

The sarcoid granuloma also produces angio- 3 tensin-converting enzyme, calcitriol, metallopep- $\stackrel{\widehat{S}}{\rightarrow}$ tidase and lysozyme, and the road to fibrosis is hastened by collagenase, type III procollagen 10 terminal peptide, hyaluronic acid and fibronectio

The chest physician's road to fibrosis would te incomplete nowadays without hoardings extolling the importance of molecular biology and macrophage-derived growth and progression factors. Do not progress along this road without first consulting an excellent topical review by Shaw. ${ }^{59}$ It is sophisticated and academic but he also has a Churchillian quality to make it readable and enjoyable. He suggests that the macrophage is the conductor of fibrotic events and plays a role via platelet-derived growth factor (PDGF) as gatekeeper in regulating the transition from inflammation to fibrosis, aided and abetted by gammainterferon (IFN- $\gamma$ ) from neighbouring lymphocytes. He suggests that in sarcoidosis there may be a conflict between the profibrotic influence of 9 IFN- $\gamma$ on macrophage PDGF gene activation, and the antifibrotic effects of combinations of IFN- $\gamma$, tumour necrosis factor and interleukin 1 on the fibroblast.

\section{Immunomodulation}

Immunotherapy has become a major branch of therapeutics, but, like Janus, it is two-headed. Immunomodulators may overcome adverse immune reactions, but they also depress immunity and lower resistance against infection. These are the twin dilemmas confronting every transplant 
team, and of particular importance to the chest physician. Corticosteroids, azathioprine, cyclophosphamide and chlorambucil are already well recognized and used worldwide. They are now being joined or supplanted by cyclosporin, FK 506, interferons, immunoglobulins and RSCD4 (Table III). The chest physician must keep abreast of their expanding use since he is called upon to deal with the immunocompromised patient with recurrent infections.

\section{Cyclosporin}

Cyclosporin (BP) or ciclosporin (WHO), derived from a Norwegian fungus, is an undecapeptide with a cyclic sequence of 11 amino acids. It is available as an oral solution, as capsules, and as an intravenous infusion. It acts by inhibiting interleukin 2 (IL-2), produced by certain cytotoxic T-helper cells, which recognize and kill target antigens, including foreign transplants. Cyclosporin is now enjoying an expanded role in the management of many autoimmune disorders, skin diseases and in ophthalmology (Figure 1). It would be used more frequently but for its nephrotoxicity and other unpleasant side effects. It is being used in South Africa with some success as a corticosteroidpotentiating agent in cryptogenic fibrosing alveolitis and progressive symptomatic interstitial lung disease of unknown aetiology. ${ }^{60}$

\section{FK 506}

Since cyclosporin is toxic, there is a continuing search for alternatives. FK 506, a Japanese discovery, is a macrolide produced by Streptomyces tsukubaensio, and in some respects is similar to erythromycin. It is much more powerful than cyclosporin in inhibiting IL-2 synthesis and the expression of IL-2 receptors, even though it is chemically unrelated. In Pittsburgh, liver transplant patients, whose plight was desperate because their liver grafts were being rejected despite conventional immunosuppression, are leading normal lives on FK 506 alone. $^{61}$ They are well without corticosteroids and azathioprine. It is now undergoing intensive clinical trials in Britain. It may be sight-saving in Behcet's disease (Ohno, personal communication).

\section{Interferons}

Human interferons are a family of proteins which activate intracellular oligo-adenylate synthetase to interfere with and inhibit viral RNA. This interference led to their name interferon. They have a wide range of antiviral and antitumour effects. Interferon is prepared by recombinant technology, and is available as a megaunit dosage for subcutaneous, intramuscular, intravenous, intrathecal and intralesional use. Trials are under way to assess its role in viral infections, malignancy and autoimmune disorders, in conjunction with other treatments. For patients with chronic granulomatous disease, interferon gamma therapy is an effective and well-tolerated treatment that reduces the frequency of serious infections. ${ }^{62}$

\section{Immunoglobulins}

Intravenous immunoglobulin preparations are now much improved compared with the outdated intramuscular preparations. It can be administered in a larger dose, minimizing the anaphylactic reactions of the old intramuscular versions. It is prepared from pooled human plasma and provides passive immune prophylaxis against hepatitis $A$, measles, rubella in pregnancy, congenital hypogammaglobulinaemia and idiopathic thrombocytopenic purpura.

When considering intravenous immunoglobulin replacement therapy it is important to distinguish and consider particularly those patients who may have near-normal immunoglobulin levels but have marked impairment in their ability to produce specific antibodies after immunisation with polysaccharide and protein antigens. These are the truly deficient persons who need replacement immunogloblin. ${ }^{63,64}$ The National Institute of Child Health has conducted a multicentre randomised doubleblind placebo (albumin)-controlled trial in 372 HIV-infected children and shown that intravenous immunoglobulin in a dose of $400 \mathrm{mg} / \mathrm{kg}$ per month was effective in significantly increasing the time free of serious bacterial infections for those children with CD4 + lymphocyte counts $<0.2 \times 10^{9}$ per litre. ${ }^{65}$

The use of intravenous immunoglobulin after bone marrow transplantation reduced the incidence and severity of interstitial pneumonia related to cytomegalovirus, sepsis and graft-versus-host disease. ${ }^{63}$ It is of value in chronic lymphocytic leukaemia but its cost may be prohibitive. ${ }^{66}$

Intravenous immunoglobulin has been effective in immunoregulatory disorders, such as immune thrombocytopenic purpura, immune neutropenia and the Kawasaki syndrome. ${ }^{64}$ It blocks the uptake of antibody-coated cells in the spleen, inhibits antibody synthesis, and inhibits inflammation. It may have a future potential use in polymyositis, Guillain-Barré syndrome, myasthenia gravis and steroid-dependent asthma. ${ }^{64}$

Intravenous immunoglobulin is recommended for systemic vasculitis. ${ }^{67}$ Following treatment there was a fall in circulating anti-neutrophil cytoplasm antibodies (ANCA) and in C-reactive protein changes and clearing of radiological charges without adverse effects. It was infused at $0.4 \mathrm{~g} / \mathrm{kg}$ per day for 5 days. 


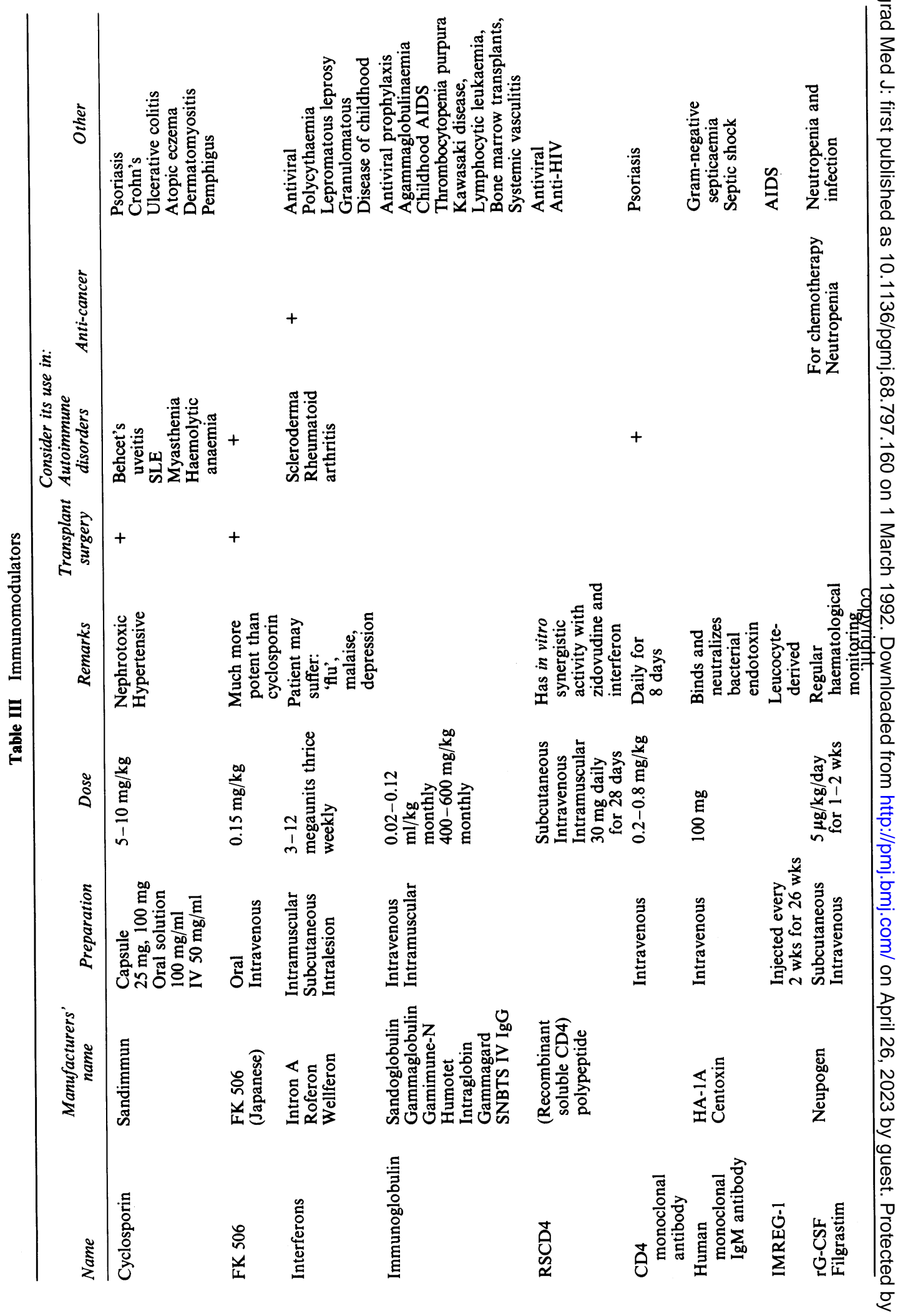




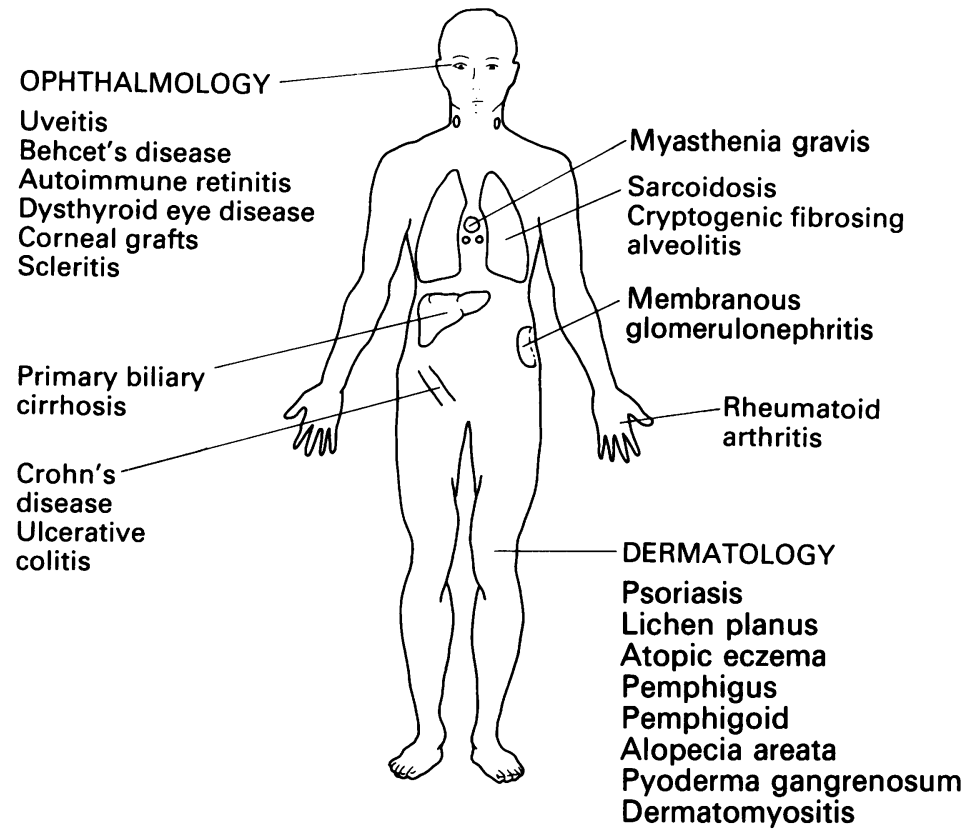

Figure 1 The expanding uses of cyclosporin.

\section{Others}

Table III also draws attention to other immunomodulators which are on the horizon and appear promising; next year they may become widely used if the cost is not prohibitive. ${ }^{68}$ They include recombinant soluble CD4 polypeptide and IMREG-1 for AIDS $;{ }^{69}$ CD4 monoclonal antibody for psoriasis; ${ }^{70}$ monoclonal antibody (HA-1A; centoxin) for Gram-negative septicaemia and septic shock:;1 and recombinant granulocyte-colony stimulating factor (rG-CSF) for neutropenia and infection in patients treated with cytotoxic chemotherapy for non-myeloid malignancy and for reduction of fever and neutropenia induced by chemotherapy in patients with small-cell lung cancer. ${ }^{72}$

\section{Other treatments}

\section{Surfactant therapy}

Neonatologists continue to find surfactant helpful, and it is now seen to be a significant advantage to administer the initial dose as prophylactic rather than as rescue therapy in very premature infants. ${ }^{73}$ It is administered into the trachea to prevent respiratory distress syndrome (RDS or hyaline membrane disease). The first such drug to be licensed in Britain is Colfosceril palmitate (Exosurf, Wellcome). Treatment has halved the overall mortality from RDS in premature infants during the first week of life, and complications such as pneumothorax are fewer. One vial of Exosurf costs $£ 314$ and up to four may be needed to treat one baby. ${ }^{74,75}$

\section{Device-related infection and disinfection}

Ten bronchoscopes that had been used on patients with AIDS were examined for microbial contamination by irrigating the suction biopsy channel with viral transport medium. Samples, tested by polymerase chain reaction, revealed HIV and also, occasionally, Pneumocystis carinii and commensal bacteria and hepatitis B virus once. Cleaning in detergent and 2 min disinfection in $2 \%$ alkaline glutaraldehyde proved effective. HIV is more resistant to glutaraldehyde in the presence of protein so detergent precleaning is essential after every bronchoscopy procedure and should not be delayed until after disinfection. ${ }^{76}$

The principal life-threatening complication in patients who have vascular catheters is septicaemia. To assess the best means of cutaneous disinfection before insertion of an intravascular catheter, a prospective study of three antiseptics was undertaken. ${ }^{77}$ The use of $2 \%$ chlorhexidine was found to be superior to $10 \%$ povidone-iodine or $70 \%$ alcohol before catheter insertion and during follow-up care. 


\section{References}

\section{Techniques}

1. James, D.G., Rizzato, G. \& Sharma, O.P. Bronchopulmonary lavage (BAL), a window of the lungs. Sarcoidosis 1992,9 (in press).

2. Menzies, R. \& Charbonneau, M,. Thoracoscopy for the diagnosis of pleural disease. Ann Int Med 1991, $114: 271$-276.

3. Wakabayashi, A., Brenner, M., Kayalah, R. et al. Thoracoscopic carbon dioxide laser treatment of bullous emphysema. Lancet 1991, 337: 881-884.

4. Chuen Lee, P. \& Hallsworth, P. Rapid viral diagnosis in perspective. $\mathrm{Br}$ Med J 1990, 300: 1413-1418.

5. Littler, E., Baylis, S.A., Zeng, Y., Conway, M.J., Mackett, M. \& Arrand, J.R. Diagnosis of nasopharyngeal carcinoma by means of recombinant Epstein-Barr virus proteins. Lancet 1991, 337: 685-689.

6. Ambroise-Thomas, P. \& Cristina, N. Results and prospects of new DNA probes and polymerase chain reaction (PCR) in toxoplasmosis. Bull Acad Natle Med 1991, 175: 39-49.

7. Wakefield, A.E., Guiver, L., Miller, R.F. \& Hopkin, J.M. DNA amplification on induced sputum samples for diagnosis of Pneumocystis carinii pneumonia. Lancet 1991, 337: $1378-1379$.

8. Brisson-Noel, A., Aznar, C., Chureau, C. et al. Diagnosis of tuberculosis by DNA amplification in clinical practice evaluation. Lancet 1991, 338: 364-366.

9. Matthews, A.W. \& Buchanan, R. A case of pulmonary veno-occlusive disease and a new bronchoscopic sign. Respir Med 1990, 84: 503-505.

10. Soler, P., Tazi, A., Basset, F.F. \& Hance, A.J. Les cellules dendritiques pulmonaires. Rev Mal Respir 1991, 8: 191-196.

11. Wernecke, K. Percutaneous biopsy of mediastinal tumours under sonographic guidance. Thorax 1991, 46: 157-159.

12. Gorge, G., Erbel, R., Schuster, S., Ge, J. \& Meyer, J. Intravascular ultrasound in diagnosis of acute pulmonary embolism. Lancet 1991, 337: 623-624.

13. Masuda, T., Shimura, S., Sasaki, H. \& Takishima, T. Surfactant aproptein-A concentration in sputum for diagnosis of pulmonary alveolar proteinosis. Lancet 1991, 337: 580-582.

\section{Wegener's Granulomatosis}

14. James, D.G. In memoriam: Dr Friedrich Wegener (1907-1990). Sarcoidosis 1991, 8: 80-81.

15. Travis, W.D., Hoffman, G.S., Leavitt, R.Y., Pass, H.I. \& Fauci, A.S. Surgical pathology of the lung in Wegener's granulomatosis. Review of 87 open lung biopsies from 67 patients. Am J Surg Pathol 1991, 15: 315-333.

16. Small, J.H., Round, A., Simpson, R.H.W. \& Ferguson, A.D. Wegener's granulomatosis simulated by a T cell lymphoma of the lung. Thorax 1991, 46: 465-466.

\section{Occupational lung disease}

17. Rom, W.N., Travis, W.D. \& Brady, A.R. Cellular and molecular basis of the asbestos-related diseases. Am Rev Respir Dis 1991, 143: 408-422.

18. Schwartz, D.A. New developments in asbestos-induced pleural disease. Chest 1991, 99: 191-198.

19. Al Jarad, N., Poulakis, N., Pearson, M.C., Rubens, M.B. \& Rudd, R.M. Assessment of asbestos-induced pleural disease by computed tomography-correlation with chest radiograph and lung function. Respir Med 1991, 85: 203-208.

20. Hosoda, Y., Ueda, A. \& Fujii, M.D. Clay dye pneumoconiosis among rush mat workers. Sem Respir Med 1991, 12: 55-57.

21. Grobbelaar, J.P. \& Bateman, E.D. Hut lung: a domestically acquired pneumoconiosis of mixed aetiology in rural women. Thorax 1991, 46: 334-340.

22. Seaton, A. Surveillance of work related and occupational respiratory disease-SWORD. Thorax 1991, 46: 548.
Papworth Hospital

23. Scott, J.P., Higenbottam, T.W., Wallwork, J. et al. Natural $\subset$ history of chronic rejection in heart-lung transplant recip- $\Rightarrow$ ients. J Heart Transplant 1990, 9: 510-515.

24. Smyth, R.L., Higenbottam, T.W., Wallwork, J. et al. Herpes simplex virus infection in heart-lung transplant recipients. Transplantation 1990, 49: 735-739.

25. Scott, J.P., Higenbottam, T.W., Clelland, C.A., Smyth, R.L. Stewart, S. \& Wallwork, J. The natural history of chronic rejection in heart-lung transplant recipients: a clinical, pathological, and physiological review of 29 long-term os survivors. Transplant Proc 1990, 22: 1474-1476.

26. Dinh-Xuan, A.T., Higenbottam, T.W., Wallwork, J. et al. Impairment of endothelium-dependent pulmonary-artery relaxation in chronic obstructive lung disease. $N$ Engl J Med 1991, 324: 1539-1547.

27. Stewart, D.J., Levy, R.D., Cernacek, P. \& Langleben, D. Increased plasma endothelin-1 in pulmonary hypertension: Marker or mediator of disease? Ann Intern Med 1991, 114: 464-469.

Tuberculosis

28. US Department of Health and Human Services. Core Curriculum on Tuberculosis. Centers for Disease Control, Atlanta, Georgia, 1991, pp. 1-40.

29. Charatan, F. Tuberculosis soars in New York. Br Med J 1991, 303: 209.

30. Monno, L., Angarano, G., Carbonara, S. et al. Emergence of drug-resistant Mycobacterium tuberculosis in HIV-infected patients. Lancet 1991, 337: 852.

31. Kochi, A. The global tuberculosis situation and the new control strategy of the World Health Organisation. Tubercg 1991, 72: 1-6.

32. Nisar, M., Williams, C.S.D. \& Davies, P.D.O. Experience tuberculosis in immigrants from Hong Kong-implications for the imminent lease back of Hong Kong. Respir Med 1991, 85: $219-222$.

33. Hong Kong Chest Service/British Medical Research Council. Controlled trial of 2,4 and 6 months of pyrazinamide in 6-month three-times-weekly regimens for smear-positive pulmonary tuberculosis, including an assessment of a combined preparation of isoniazid, rifampin and pyrazinamide. Am Rev Respir Dis 1991, 143: 700-705.

34. Singapore Tuberculosis Service/British Medical Research Council. Assessment of a daily combined preparation of isoniazid, rifampin and pyrazinamide in a controlled trial of three 6-month regimens for smear-positive pulmonary tuberculosis. Am Rev Respir Dis 1991, 143: 707-712.

35. Horsburgh, C.R. Mycobacterium avium complex infection in the acquired immunodeficiency syndrome. $N$ Engl $J$ Med 1991, 324: 1332-1338.

Fungal infections

36. Spellar, D.C.E. \& Warnock, D.W. Developments in the management of fungal infection. $J$ Antimicrob Chemother 1991, 28 (Suppl A): 1-103.

37. Walsh, T.J., Hathorn, J.W., Sobel, J.D. et al. Detection of circulating candida enolase by immunoassay in patients with cancer and invasive candidiasis. $N$ Engl J Med 1991, 324: 1026-1031.

38. British Society for Antimicrobial Chemotherapy Working Party. Laboratory monitoring of antifungal chemotherapy. Lancet 1991, 337: 1577-1580.

\section{BOOP}

39. Bellomo, R., Finlay, M., McLaughlin, P. \& Tai, E. Clinical spectrum of cryptogenic organising pneumonia. Thorax 1991, 45: 554-558.

40. Woodhead, M.A. \& Dubois, R.M. Bronchiolitis obliterans, cryptogenic organising pneumonitis and BOOP. Respir Med 1991, 85: 177-178. 
41. Cordier, J.F., Loire, R. \& Peyrol, S. Bronchiolitis obliterans organising pneumonia (BOOP). Rev Mal Respir 1991, 8: $139-152$.

42. Rees, J.H., Woodhead, M.A., Sheppard, M.N. \& DuBois, R.M. Rheumatoid arthritis and cryptogenic organising pneumonitis. Respir Med 1991, 85: 243-246.

43. Perez, T., Dansin, E., Wallaert, B. \& Tonnel, A.B. Manifestations pleuro-pulmonaires de la polyarthrite rhumatoide. Rev Mal Respir 1991, 8: 169-189.

44. Case Records of the Massachusetts General Hospital. Case 17-1991. N Engl J Med 1991, 324: 1195-1205.

45. Geddes, D.M. BOOP and COP. Thorax 1991, 46: 545- 547.

\section{New syndrome}

46. Marguerie, G., Bunn, C.C., Beynon, H.L.C. et al. Polymyositis, pulmonary fibrosis and autoantibodies to aminoacyl-tRNA synthetase enzymes. $Q J$ Med 1990, NS 77: 282: 1019-1038.

\section{Neoplasia}

47. Idhe, D.C. \& Minna, J.D. Non small cell lung cancer Part 1: Biology, diagnosis and staging. Current Problems in Cancer. Mosby Year Book 1991, XV: 63-104.

48. Gosney, M.A., Gosney, J.R. \& Lye, M. Plasma growth hormone and digital clubbing in carcinoma of the bronchus. Thorax 1990, 45: 545-547.

49. Lee, J.S., Ro, J.Y., Sahin, A.A. et al. Expression of bloodgroup antigen $\mathbf{A}-\mathbf{a}$ favourable prognostic factor in nonsmall cell lung cancer. $N$ Engl J Med 1991, 324: 1084-1090.

50. Donnadieu, N., Paesmans, M. \& Sculier, J.P. Chimiotherapie des cancers bronchiques non à petites cellules. Meta-analyse de la littérature en fonction de l'extension de la maladie. Rev Mal Respir 1991, 8: 197-204.

51. Murren, J.R., Buzaid, A.C. \& Hait, W.A. Critical analysis of neoadjuvant therapy for Stage IIIa non-small cell lung cancer. Am Rev Respir Dis 1991, 143: 889-894.

52. Beral, V., Peterman, T., Berkelman, R. \& Jaffe, H. AIDSassociated non-Hodgkin lymphoma. Lancet 1991, 337: 805-809.

53. Henry-Amar, M., Friedman, S., Hayat, M. et al. Erythrocyte sedimentation rate predicts early relapse and survival in early-stage Hodgkin disease. Ann Intern Med 1991, 114: $361-365$.

54. Litam, P., Swan, F., Cabanillas, F. et al. Prognostic value of serum $\beta$ - 2 microglobulin in low-grade lymphoma. Ann Intern Med 1991, 114: 855-860.

\section{Sarcoidosis}

55. Proceedings of the Twelfth World Congress on Sarcoidosis, Kyoto, Japan. Sarcoidosis 1992 (Suppl).

56. Miyachi, A., Noda, M., Ina, Y. et al. Tumour necrosis factor in granulomatous lung disease. Sarcoidosis 1989, 6: 80.

57. Foley, N.M., Millar, A.B., Meager, A., Johnson, N.M. \& Rook, G.A.W. Tumour necrosis factor production by alveolar macrophages in pulmonary sarcoidosis and tuberculosis. Sarcoidosis 1992 (in press).

58. James, D.G. What makes granulomas tick? Thorax 1991, 46: 734-736.

59. Shaw, R.J. The role of lung macrophages at the interface between chronic inflammation and fibrosis. Respir Med 1991, 85: $267-273$.

\section{Immunomodulators}

60. Moolman, J.A., Bardin, P.G., Rossouw, D.J. \& Joubert, J.W. Cyclosporin as a treatment for interstitial lung disease of unknown aetiology. Thorax 1991, 46: 592-595.

61. Starzl, T.E., Todo, S., Fung, J. et al. FK 506 for liver, kidneys and pancreas transplantation. Lancet 1989, ii: 1000-1004.

62. International Chronic Granulomatous Disease Co-operative Study Group. A controlled trial of interferon gamma to prevent infection in chronic granulomatous disease. $N$ Engl $J$ Med 1991, 324: 509-516.

63. Buckley, R.H. \& Schiff, R.I. The use of immune globulin in immunodeficiency diseases. $N$ Engl $J$ Med 1991, 325: 110-117.

64. Stiehm, E.R. New uses for intravenous immune globulin. $N$ Engl J Med 1991, 325: 123-125.

65. The National Institute of Child Health and Human Development Intravenous Immunoglobulin Study Group. Intravenous immune globulin for the prevention of bacterial infections in children with symptomatic human immunodeficiency virus infection. $N$ Engl J Med 1991, 325: 73-80.

66. Weeks, J.C., Tierney, M.R. \& Weinstein, M.C. Costs effectiveness of prophylactic intravenous immune globulin in chronic lymphocytic leukaemia. $N$ Engl J Med 1991, 325: 81-86.

67. Jayne, D.R.W., Davies, M.J., Fox, C.J.V., Black, C.M. \& Lockwood, C.M. Treatment of systemic vasculitis with pooled intravenous immunoglobulin. Lancet 1991, 337: 1137-1139.

68. Taylor, D. Centoxin-birth of a budgetbuster. Br Med J 1991, 302: 1229.

69. Gottlieb, M.S., Zackin, R.A., Fiala, M. et al. Response to treatment with the leukocyte-derived immunomodulator IMREG-1 in immunocompromised patients with AIDSrelated complex. Ann Intern Med 1991, 115: 84-91.

70. Prinz, J., Braun-Falco, O., Daddona, P. et al. Chimaeric CD4 monoclonal antibody in treatment of generalised pustular psoriasis. Lancet 1991, 338: 320-321.

71. Ziegler, E.J., Fisher, C.J., Sprung, C.L. et al. Treatment of gram-negative bacteremia and septic shock with HA-1A human monoclonal antibody against endotoxin. A randomized, double-blind, placebo-controlled trial. $N$ Engl J Med 1991, 324: 429-436.

72. Crawford, J., Ozer, H., Stoller, R. et al. Reduction by granulocyte colony-stimulating factor of fever and neutropenia induced by chemotherapy in patients with small-cell lung cancer. $N$ Engl J Med 1991, 325: 164-170.

\section{Other treatments}

73. Kendig, J.W., Notter, R.H., Cox, C. et al. A comparison of surfactant as immediate prophylaxis and as rescue therapy in newborns of less than 30 weeks gestation. N Engl J Med 1991, 324: 865-871.

74. Avery, M.E. \& Merritt, T.A. Surfactant-replacement therapy. $N$ Engl J Med 1991, 324: 910-911.

75. Anonymous. Colfosceril palmitate (Exosurf)-artificial surfactant for immature lungs. Drug Therapeut Bull 1991, 29: 46.

\section{Device-related infection and disinfection}

76. Hanson, P.J.V., Gor, D., Clarke, J.R. et al. Recovery of the human immunodeficiency virus from fibreoptic bronchoscopes. Thorax 1991, 46: 410-412.

77. Maki, D.G., Ringer, M. \& Alvarado, C.J. Prospective randomised trial of povidone-iodine, alcohol, and chlorhexidine for prevention of infection associated with central venous and arterial catheters. Lancet 1991, 338: 339-343. 